\title{
The Relationship between Materials Science and the Fourth Industrial Revolution
}

\author{
Zoltán KOLOZSVÁRY
}

SC Plasmaterm SA; Sapientia "Sapientia” Hungarian University of Transylvania, Târgu-Mureș, Romania. zoltan.kolozsvary@plasmaterm.ro

\begin{abstract}
There has been a spectacular and extremely fast development in all areas of materials science. This development is driven by science and technology, yet a time gap may be observed between the progress of technology that drives the fourth industrial revolu-tion, and its acceptance in society. Our task is to learn how a balance may be achieved between rapid technological development and societal acceptance.
\end{abstract}

Keywords: materials science, fourth industrial revolution, technology, society, time gap, additive manufacturing, surface treatment, conflict risk materials.

\section{Introduction}

Throughout the history of mankind, there has been a characteristically strong interdependence between the development of technology, and the development of society. The time gap between these two depends on communication and primarily on its speed.

The easier and faster the communication, the faster and stronger is the social reaction to it. After centuries of "accumulation”, the 20 century has accelerated this spiral, generating even more impetus in the first decades of the $21^{\text {st. }}$ century.

The last two decades of the twentieth century and the first two of the twenty first have remodelled the concept of science and technology. A pronounced time gap has appeared between changes in industry and their social interpretation.

The beginning of the $21^{\text {st }}$ century ,attacked” human society with unprecedented speed. The potential of data processing and data transmission increased spectacularly and- as a consequencethe borders in almost every area of daily life expanded significantly, even if not always to the benefit of the society.

Today it seems to be a common place saying that „the twentieth century was the century of physics", and this cannot be denied; its effects are sensed (or sometimes suffered) even today. No doubt, this is a very sophisticated question as the interdependence between technology and society has always been critical, and determining this interdependence has become even more relevant with the increasing data processing and data transmission as Figure 1. demonstrates.

„... the part and the whole...” as Werner Heisenberg formulated was never more actual than it is today!

The unprecedented rate of development of science and technology is digging deeper and deeper into materials and brings to the surface their ever-smaller details.

... however all these are only „details” which may bring a significant development for human being and society, if we do not forget the „whole”. The goal of this paper is to outline a short, synthetic summary on „the whole” ... approaching it from the point of view of materials science.

\section{The social responsibility of material scientists regarding material use}

The Figure 1. schematically outlines the interdependencies of science and technology and the society, emphasizing the today dominant triangle which - in fact - is propelled by materials science.

If we try to briefly outline the main directions, these should be summarized as:

- Ever increasing expectations on performance, with a simultaneous reduction of the production costs and energy needs, whilst not neglecting the reduced environmental hazard. 


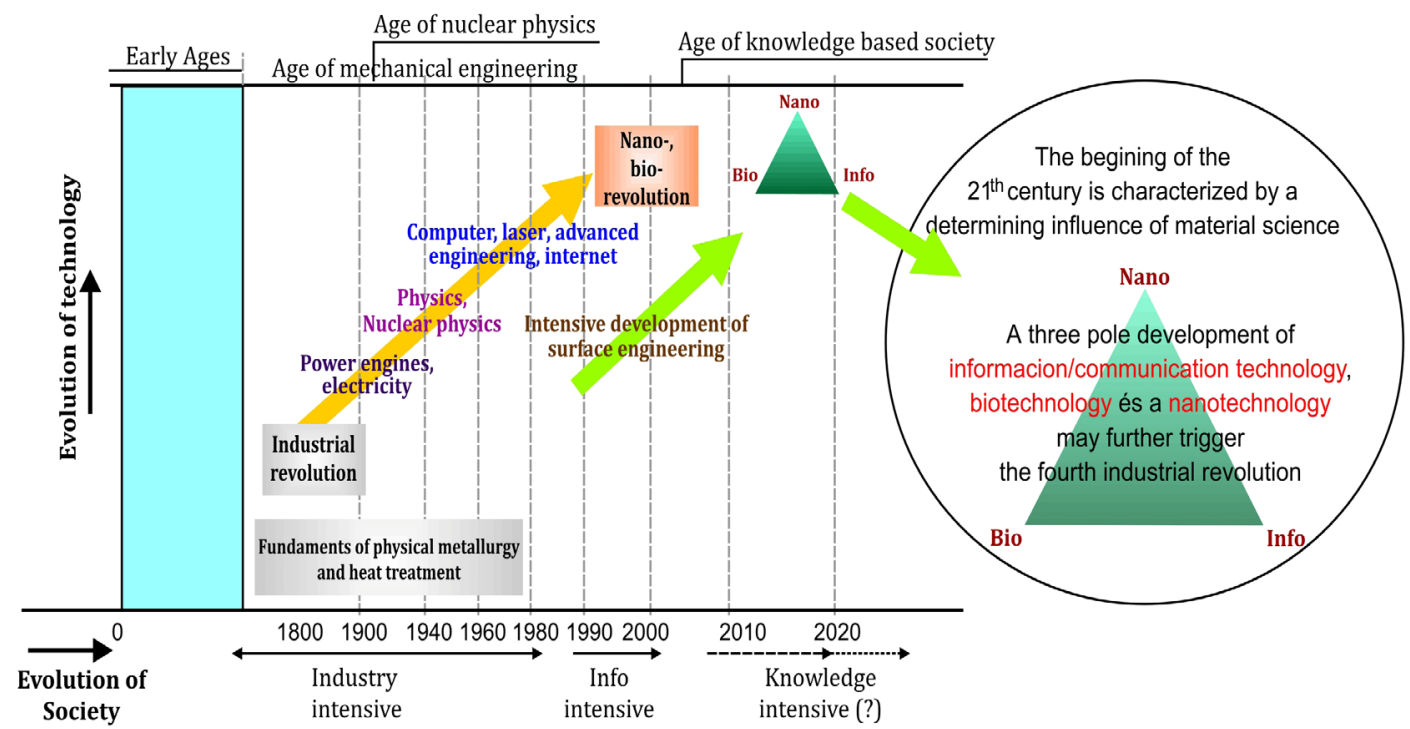

Figure 1. Interdependence between science, technology and society

- The spectacular development of investigating methods and instrumentation has opened new possibilities to learn, experiment and develop new materials, often with almost surprising properties. (Just one example: new gold-platinum alloy with unprecedented wear resistance and a hardness comparable with that of the diamond) [1].

Though new materials appear almost at a daily rate, development is also very significant in the field of those materials considered „classic”.

The specific material consumption is decreasing in almost any industrial field but the global material need is spectacularly increasing. The world is slowly beginning to suffer a material crisis. Technical materials are becoming more and more diverse, there are always new expectations as functions of new technologies, a good example for this is 3D printing [2]. On approaching the topic of critical materials it's enough to look at the Periodic Table of Elements

A critically important field of the global economy is energy production, and even more, energy storage. The efficiency of the systems and mechanisms largely depends on the properties of the used materials. Solar panels, wind turbines and hydrogen fuel cells all generate extreme expectations from materials. Although it can seem like the „usual” materials that have been subjected to special surface improving treatments still form the majority, there are more and more fields where less conventional materials, alloys or even non-metals are used. Going back to the field of our research let's consider a few principles that are important for those involved in scientific research, development and innovation, regarding the economic use of materials.

- The rational use of materials has to start from the design.

- The total life-cycle of materials has to increase, and recycling has to be considered, whenever possible.

- Technologies with minimum waste have to be developed.

- In order to increase the useful lifecycle of products, efficient heat treating and surface engineering technologies have to be developed.

- For reducing the waste resulting from design or technology, computer modelling and simulation have to be applied on a large scale.

- It is also important to consider the problem of critical materials, the "conflict mineral risk" It is enough to claim just a few of these: cobalt, titanium, gold, platinum, tantalum, tungsten, vanadium, neodymium, lithium, rare earth metals etc. [3]. In the case of critical materials or endangered materials alternative solutions have to be developed by advanced research and development procedures.

\section{A few important considerations on optimal use of materials}

The principles of material use that consider the future too are increasingly present in the work- 
ings of society, but there are more and more urgent signs of a need for a long term responsible thinking. That's why I think it's important to evidentiate some important principles regarding material use:

- On a short run the need for developing new materials will not effectively influence the interest for the materials in general use on a large scale.

- Iron alloys will, in future, also be the most important engineering materials, however their properties will undergo significant modifications.

- Aluminum and titanium alloys will increasingly become the focus of attention, especially for transport vehicles.

- There is an ever-increasing interest in composites but significant changes are to be expected concerning their properties.

- The increasing application of graphite in its different forms (graphen, aerografite, fulleren a.s.o) seems very interesting... It seems a rational question should be: after the Iron Age, is the „carbon age” coming ...?

- In order to reduce energy needs -primarily for transport vehicles- there is an increasing interest in developing light weight structures without reducing their efficiency. This should be a result first of all of a rational development but the use of lightweight materials becomes more and more the focus of attention. In this respect aluminum is the focus of attention, - even if the energy need for its metallurgy is considerable and difficulties appear also in recycling, which means the "total lifecycle" is more limited compared to the steel [4].

- The increasing interest in light alloys in the vehicle industry is also orienting interest towards new plastic deformation- and surface engineering technologies (this certainly is true also for titanium alloys).

Following this line of "philosophy" a brief look at Figure 2. should be of interest. It illustrates the changes in uses of materials for the carrosseries of transport vehicles. The interest in composites and light alloys versus that of steel seems evident.

The increasing interest in 3D printing in the most diverse application areas is also evident, including aerospace and medical instrumentation.

In additive manufacturing, the structure of materials may be well projected supporting specific properties which has a particular importance [5, 6].

\section{Fields of key importance in industry}

When additive manufacturing is considered, two industrial areas are of particular importance: the vehicle industry and energy generation/storage. In this respect development is really spectacular, with a special emphasis on metal additive manufacturing.



Figure 2. Changes in material use in the automotive industry 
For the moment the problems connected to metallurgy of material structure, like surface modification technologies, are very little known. Significant differences appear in the metallurgy function of applied procedures. Fast solidification, oriented cooling and changes in the structure due to repeated heating and cooling, all strongly influence the microstructure of the material. All these processes are very little known at present, as these are radically different from those associated with conventional processes [7].

The procedures for modifications in the structure of bulk material and those of the surface are very poorly known and form a totally new area which -until now- has been very little studied and investigated (that is to say that surface treatments i.e. Figure 3.) form a fundamentqally new domain, little known or studied presently. [8].

It would be a mistake not to consider the preoccupation concerning the study of materials produced by nature, and we have to learn from the „mother nature”... According to T. S. Sudarshan: nature is ,a warehouse of futuristic materials where the impossible becomes possible" [9].

- Nature offers simple solutions with degradable materials and using available resources (Water, air, minerals, organics-proteins, sunlight).

- Uses only the energy needed and disposes of the rest making it a model of sustainability.

- No pollution - filled with optimization!

A strong increase of interest in bio-inspired and nano technology-based surface modification may also be observed [10]. New surface treatment technologies appear constantly (Figure 4.) to which I give only two examples:

- In order to reduce the friction of contact surfaces their structure is modified, imitating the shark-skin structure.

- Developing different nano-surface structures (imitating the lotus leaf effect).

These are just a few examples but the possible applications are almost endless!

Concerning new developments we should never forget the danger of ,collateral damage”.

In case of materials science little attention is paid to the total lifecycle of materials, including recycling and annihilation.... In the $20^{\text {th }}$ century the ever increasing application of polymers was characteristic and this trend is even more powerful at the beginning of this century. However, no attention has been given to the problem of ,afterlife" of the materials.
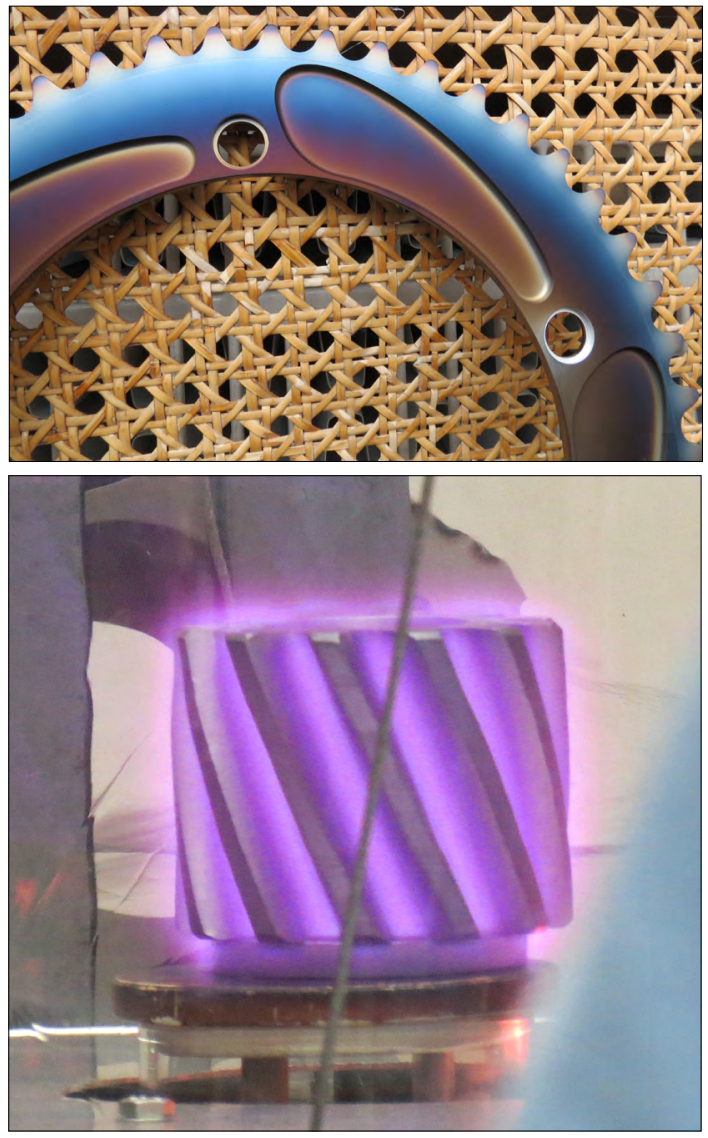

Figure 3. Aluminium alloy gear with highly abrasion-resistant PVD coating for racing bicycles (top) and steel gear in the plasma nitriding process (bottom)

\section{Industrial development in the pre- sent and the societal challenges for ma- terial science}

It is well known that the natural decomposition of synthetic materials is a slow process taking centuries, but - unfortunately - the tremendous damage they may cause in the natural environment and the deleterious consequences also on the food chain has only recently become obvious.

The increasing population of the Earth - that needs feeding - , the difficulties of providing drinking water show us that there's hardly any time left for us to continue our waste of materials.

It is increasingly evident that human society has arrived at a point where it is not satisfactory to talk about sustainable development only in terms of political slogans, but efficient actions have to be initiated. A self-evident question has to be put: what is the real driving force of industrial and so- 


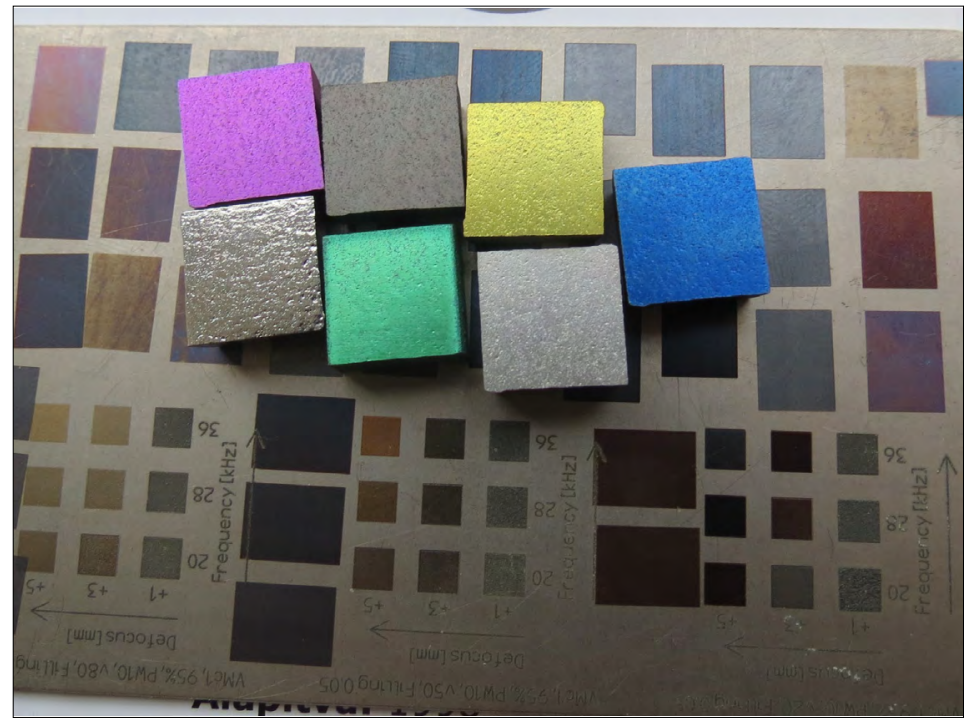

Figure 4. Titanium implant material colored by anodizing on a titanium alloy plate colored by laser engraving

cial development (?)... we like to say: the driving force of development is the concern for energy and environment.... but in fact, we do very little to prevent damage to the environment and to prevent the unlimited waste of materials.....as a consequence it is self-evident what the real driver of industrial and economic development is: instant and limitless profit!

Materials science is one of the fundamental factors provoking the fourth industrial revolution. It has a complex and very pronounced influence on the society and on its future development, but development and environment are not separable challenges.

Considering development, we should never forget that there is a system of extremely complex and sophisticated interactions covering economic, technological, ecologic and societal aspects.

Materials science has to find scientific-technological solutions for preventing damage to the environment and to restore its healthy status. [11]. Materials science has to find scientific-technological solutions for preventing damage to the environment and to restore its healthy status.

We should no longer say: "this is not the task of materials science" ... and that what happens to the materials in their "afterlife" is not our concern!

It is important how we see the problem of energy and environment... each coin has two sides... The problem of energy is the focus of attention in: - Global economy

- Research and development
- Industry

The environment is the concern of

- People in general

- Responsible (?) politicians

- Mass media

... but at the end of the day that is just the same coin!....[12].

In this article I intended to present those most important realizations that I came to while working in the field of materials science and technology, regarding the parallel development of industry and society. As a final conclusion and in full agreement with it I quote a thought by professor Jianguo Liu from Michigan State University:

“....it is time to integrate all disciplines for fundamental discoveries and synergetic solutions because of increasingly connected world challenges.

Disciplinary approaches to crises like air pollution, biodiversity loss, climate change, food insecurity and energy and water shortages are not only ineffective but also making many of these crises worse because of counterproductive interactions and unintended consequences” [13]

\section{References}

[1] Curry J. F., Babuska T. F., Furnish T. A., Ping Lu, Adams D. P., Kustas A. B., Nation B. L., Dugger M. T., Chandross M., Clark B. G., Boyce B. L., Schuh C. A., Argibay N.: Achieving ultralow wear with stable nanocrystalline metals. Advanced Materials, 30/32. (2018) 1802026, 1-7.

https://doi.org/10.1002/adma.201802026 
[2] Ashby M.: University of Cambridge and Granta Design, Cambridge, 2014.

[3] Dodd-Frank Wall Street Reform and Consumer Protection Act. Public Law 111-203, July 21, 2010, 1375-2223.

https://www.congress.gov/111/plaws/publ203/ PLAW-111publ203.pdf

[4] Tisza M., Czinege I.: Comparative study of the application of steels and aluminium in lightweight production of automotive parts. International Journal of Lightweight Materials and Manufacture, 1/4. (2018) 229-238.

https://doi.org/10.1016/j.ijlmm.2018.09.001

[5] Frazier W. E.: Metal additive manufacturing: A review. Journal of Materials Engineering and Performance, 23/6. (2014) 1917-1928.

https://doi.org/10.1007/s11665-014-0958-z

[6] Sandvik invests in metal powder plant. Heat processing, 2. (2018) 14.

[7] Kolozsvary Z.: Surface engineering: its limits for engineering applications. IFHTSE $19^{\text {th }}$ International Congress, Glasgow, 2011.
[8] Kolozsvary Z.: Surface engineering: a bridge between 'avant-garde' and conventional materials and technologies. 20. Congress of IFHTSE, Beijing, 2012.

[9] Sudarshan T. S.: Emulate to innovate. SMT 31 The 31st Edition of the International Conference on Surface Modification Technologies, July 5-7, 2017, Mons, Belgium.

[10] Fan Xia, Lei Jiang: Bio-inspired, smart, multiscale interfacial materials. Advanced Materials, 20/15. (2008) 2842-2858. https://doi.org/10.1002/adma.200800836

[11] Techniques could create better material, design in high consequence uses. ScienceDaily, September 24, 2015.

www.sciencedaily.com/releases/2015/09/15092 4084023.htm

[12] Cantonwine P. E.: Educating materials engineers. Advanced Materials and Processes, March 2006, 51-53.

[13] World's challenges demand science changes. ScienceDaily, February 26, 2015.

https://www.sciencedaily.com/releases/2015/02/ 150226144903.htm 